\title{
Cilnidipine, An L-/N-Type Calcium Channel Blocker, Changes the Circulating Angiotensin - (1-7)/Angiotensin II Ratio
}

\author{
Shizuka Aritomi ${ }^{1 *}$, Kazumi Niinuma ${ }^{1}$, Mai Kawakami ${ }^{1}$, Tarou Nakamura1 ${ }^{1}$,omoyuki Konda ${ }^{1}$, Makoto Shiozaki ${ }^{1}$ and Michihiro Yoshimura ${ }^{2}$
}

${ }^{1}$ Research Center, Ajinomoto Pharmaceuticals, Kanagawa, Japan

${ }^{2}$ Division of Cardiology, Department of Internal Medicine, The Jikei University School of Medicine, Tokyo, Japan

\begin{abstract}
Objective: It is well known that cilnidipine, which is a L/N-type calcium channel blocker (CCB), has Angiotensin (Ang) II lowering effects that result in organ protective effects compared to L-type CCBs. However, a recent study indicated that angiotensin-converting enzyme (ACE) 2, which is expressed in the heart and kidney, metabolizes Ang II to Ang-(1-7), which is a peptide that has organ-protective effects. In this study, we compared the effects of the L-/N-type CCB cilnidipine and the L-type CCB amlodipine on plasma Ang peptides in a rat hypertensive model.
\end{abstract}

Methods: Eight-week-old Sprague-Dawley rats were administered a chronic infusion of Ang II through an osmotic mini-pump, along with vehicle, cilnidipine, or amlodipine for 28 days, and the plasma renin-angiotensin-aldosterone system (RAAS) levels were examined.

Results: Cilnidipine and amlodipine exerted equivalent antihypertensive effects. As for Ang peptides, amlodipine induced increases in Ang I levels and decreases in Ang-(1-7)/Ang I ratios. However, the cilnidipine group showed significantly higher Ang-(1-7)/Ang II ratios than the control group. As for gene expression, amlodipine significantly decreased the ratio of ACE2/ACE. Moreover, only cilnidipine treatment resulted in a significant reduction in cardiac fibrosis.

Conclusions: The results of the present study demonstrate that the L-/N-type CCB cilnidipine changed the balance between Ang-(1-7) and Ang II and increased the proportion of Ang-(1-7). Taken together, these results suggest that the suppressing the expansion of the area of cardiac fibrosis of cilnidipine is due to increases in the activity of the ACE2Ang-(1-7) arm.

Keywords: Cilnidipine; Angiotensin-(1-7); Fibrosis

\section{Introduction}

Angiotensin-converting enzyme (ACE) 2 is a newly recognized ACE homolog within the renin-angiotensin system (RAS) that is produced and secreted by a variety of cell types [1]. Although ACE2 may act on several substrates, it exhibits high catalytic efficiency, specifically for the hydrolysis of angiotensin (Ang) II to the vasodilator and growth inhibitor heptapeptide Ang-(1-7) [2-4]. Previous reports have suggested that Ang-(1-7) exerts vasodilatory effects through a combination of ACE inhibition and angiotensin type 1 receptor (AT1R) blockade [5]. Taken together, these studies suggest that the regulation of Ang-(1-7) production by ACE2 may be an important component of not only blood pressure control but also vascular remodeling because Ang-(1-7) opposes the actions of Ang II in both cases [3]. According to this novel concept, the final functional effects of the RAS may reflect a balance between the ACE-Ang II-AT1R arm and the ACE2-Ang-(1-7)Mas receptor arm [6-8].

Cilnidipine, which is an N-type, as well as an L-type, calcium channel blocker (CCB) [9], has a variety of unique actions that are mediated by its $\mathrm{N}$-type calcium channel-blocking actions, including the suppression of sympathetic nervous activity [10-12]. We found in a previous study that cilnidipine causes no reflexive increases in plasma renin activity (PRA) and Ang II levels $[13,14]$, while the L-type CCB amlodipine causes an increase in the activity of RAS because of its antihypertensive actions [15]. Furthermore, cilnidipine suppresses Ang II receptor blocker (ARB)-induced increases in the activity of the RAS by the blockade of the N-type calcium channel [16] and exert an organprotective action when given concomitantly with an ARB [17]. Indeed, a clinical trial that compared the CCBs cilnidipine and amlodipine in combination with a RAS inhibitor showed that cilnidipine was superior to amlodipine in preventing RAS activation [18] and the progression of proteinuria in hypertensive patients [19]. However, it has never been examined if cilnidipine can control the balance between the ACE-Ang II-AT1R arm and the ACE2-Ang-(1-7)-Mas receptor arm.

In this study, therefore, we investigated the effects of the L-/Ntype CCB cilnidipine on the Ang-(1-7)/Ang II balance in hypertensive rats in comparison with the L-type CCB amlodipine in order to verify whether cilnidipine can change the balance between the 2 arms from harmful to protective.

\section{Materials and Methods}

\section{Animals}

All experiments were conducted with the approval of the animal ethics committee of Ajinomoto Co., Inc. Seven-week-old male Sprague-Dawley rats were purchased from Japan SLC, Inc (Shizuoka, Japan). The rats were given standard laboratory chow (CRF-1, Charles River Japan, Kanagawa, Japan) and tap water ad libitum throughout the experimental period.

*Corresponding author: Shizuka Aritomi, Research Center, Ajinomoto Pharmaceuticals, Kanagawa, Japan, E-mail: shizuka_aritomi@ajinomoto.com

Received December 22, 2011; Accepted January 22, 2012; Published January 26, 2012

Citation: Aritomi S, Niinuma K, Kawakami M, Nakamura T, Konda T, et al. (2012) Cilnidipine, An L-/N-Type Calcium Channel Blocker, Changes the Circulating Angiotensin-(1-7)/Angiotensin II Ratio. J Hypertens 1:102. doi:10.4172/21671095.1000102

Copyright: @ 2012 Aritomi S, et al. This is an open-access article distributed unde the terms of the Creative Commons Attribution License, which permits unrestricted use, distribution, and reproduction in any medium, provided the original author and source are credited. 


\section{Drugs}

Ang II was purchased from Peptide Institute, Inc (Osaka, Japan). The L-/N-type CCB cilnidipine (Ajinomoto Co., Inc., Tokyo, Japan) and the L-type CCB amlodipine (Lek Co. Inc., Kolodvorska, Slovenia) were used as antihypertensive drugs. Each antihypertensive drug was initially dissolved in $0.5 \%$ hydroxypropyl methylcellulose (HMC; Sigma-Aldrich, St. Louis, MO, USA).

\section{Experimental protocols}

While the 8-week-old experimental animals were under pentobarbital anesthesia $(50 \mathrm{mg} / \mathrm{kg}$, i.p., Abbott japan Co., Ltd., Tokyo, Japan), an incision was made in the back of the neck region, and an osmotic minipump (Alzet model 2ML4; Alza, Palo Alto, CA) filled with $\mathrm{Val}^{5}$-Ang II (800 $\left.\mathrm{ng} / \mathrm{kg} / \mathrm{min}\right)$ was inserted [20] for inducing hypertension. Because endogenous Ang II (Ile ${ }^{5}$-Ang II) and exogenous Ang II ( $\mathrm{Val}^{5}$-Ang II) can be separated by liquid chromatography-tandem mass spectrometry (LC-MS), this substitution approach enabled the determination of the relative contributions of exogenously infused $\mathrm{Val}^{5}$-Ang II vs the endogenously formed $\mathrm{Ile}^{5}$-Ang II to the increased levels of plasma Ang peptides [21]. On Day 1 after the operation, the rats were divided into the following 3 groups that had the same mean systolic blood pressure (SBP) and mean body weight: a control group (0.5\% HMC, per os [p.o.]), a cilnidipine group (cilnidipine, 3 or $10 \mathrm{mg} /$ $\mathrm{kg}$ /day p.o.), and an amlodipine group (amlodipine, 3 or $10 \mathrm{mg} / \mathrm{kg} / \mathrm{day}$ p.o.) for 4 weeks, and plasma RAAS levels were examined. An osmotic minipump filled with saline was also inserted in rats of the normal control group, which were given $0.5 \%$ HPMC orally for 4 weeks.

\section{Blood Pressure, and heart rate measurement}

SBP was measured every week by the tail cuff method with a Softron BP-98A (Softron Co., Ltd., Tokyo, Japan). Rats were introduced into a plastic wire holder and placed in a thermostatically warmed tube that was maintained at $34-36^{\circ} \mathrm{C}$ during the measurements. The mean of 3 measurements was determined for each animal after their acclimation to the environment.

\section{Measurement of the renin-angiotensin-aldosterone system (RAAS) and catecholamines}

To measure these biochemical parameters, the blood samples were centrifuged $(1000 \mathrm{~g})$ for $15 \mathrm{~min}$, and the plasma samples were stored at $-80^{\circ} \mathrm{C}$ until the analyses. PRA was determined using a radioimmunoassay system (Yamasa Shoyu Co. Ltd., Chiba, Japan), and the Ang peptides were purified by solid-phase extraction and measured by LC-MS in order to separate endogenous Ang peptides from exogenous Ang peptides [20]. The plasma aldosterone levels were measured with an enzyme immunoassay system (Cayman Chemical, Ann Arbor, MI). Catecholamine levels in the plasma were measured with high-performance liquid chromatography.

\section{Histological studies}

The myocardium was fixed in $10 \%$ formalin and stained with hematoxylin and eosin or Masson's trichrome in order to determine the myocyte cross-sectional dimensions and interstitial fibrosis. Analyses of the slides were performed in a blinded fashion with a light microscope (BX50, Olympus corporation, Tokyo, Japan), and the relative volume occupied by each element of the ventricle (myocardial fibers and fibrous tissue) was measured with a special ocular apparatus (WinROOF ver 3.5, MITANI, Fukui, Japan).

\section{Quantitative real-time reverse transcription-polymerase chain reaction (qRT-PCR)}

Total RNA was isolated from the heart using the RNeasy Mini kit (QIAGEN GmbH, Hilden, Germany). One microgram of total RNA was reverse transcribed with SuperScript III (Invitrogen, Carlsbad, CA, USA). qRT-PCR was performed with a 7500 Real-Time PCR System with SYBR Green Master Mix (Applied Biosystems, Foster City, CA, USA) and sequence-specific primers. The primer sequences employed were as follows: ACE2 (forward primer, 5'-GCCAGGAGATGACCGGAAA-3'; reverse primer, 5'-CTGAAGTCTCCATGTCCCAGATC-3'), ACE (forward primer, 5'-CGAATGTGCCATACATCAGG-3'; reverse primer, 5'-CCTGCTTCCTTGGATTGGTA-3'), brain natriureticpeptide(BNP) (forward primer, 5'-TGGGCAGAAGATAGACCGGA-3'; reverse primer, 5'-ACAACCTCAGCCCGTCACAG-3'), angiotensinogen (forward primer, 5'-CAGCACGACTTCCTGACTTG-3'; reverse primer, 5'-GGTAGACAGCTTGGCCTGAG-3'), and $\beta$-actin (forward primer, 5'-GAGGACCAGGTTGTCTCCTG-3'; reverse primer, 5'-ATGTAGGCCATGAGGTCCAC-3'). All mRNA levels were normalized to $\beta$-actin expression levels.

\section{Statistical analysis}

Data were expressed as means \pm SEM. One-way ANOVA, which was followed by Dunnett's multiple range test, was used to assess the differences between the control group and the CCB-treated groups. Parameters in the control group and the normal group were compared by unpaired $t$-tests. Differences were considered statistically significant when the probability values were less than 0.05 .

\section{Results}

\section{SBP, and heart rate}

SBP in the control group was significantly higher than that in the normal group at the first and last days' Ang II-infusion $(P<0.001$, and $\mathrm{P}<0.05$ Figure $1 \mathrm{~A})$. The antihypertensive effects were comparable among the Ang-II infused groups, with the CCB treatment groups showing significantly lower SBP levels than the control group at the first and last days' Ang II-infusion $(P<0.001$, and $\mathrm{P}<0.05$ Figure $1 \mathrm{~A})$. As for heart rate, there was no significantly different between the control group and the normal group during the examination (Figure 1B). And on the other hand, CCB treatment significantly elevated heart rate in comparison with the control group at the first days' Ang IIinfusion $(P<0.01$, Figure $1 \mathrm{~B})$.

\section{Body weight, and organ weight}

The body weight in the control group was significantly lower than that in the normal group $(P<0.01$, Table 1$)$. The antihypertensive effects were comparable among the Ang-II infused groups, with the CCB treatment groups showing significantly lower SBP levels than the control group $(P<0.05$ each, Table 1$)$. The blood urea nitrogen levels in the cilnidipine group were significantly lower than those in the control group $(P<0.05$, Table 1$)$. However, there were no differences in the other biochemical indexes.

\section{Production of angiotensins}

There was no difference in plasma exogenous Ang II levels among the Ang II-infused groups (data not shown). As for the endogenous Ang peptides, only the amlodipine group showed higher Ang I levels than the control group $(737.3 \pm 54.0 \mathrm{pM}$ vs $443.9 \pm 42.0 \mathrm{pM}, P<0.05$; Figure 2A). There was no difference in Ang II and Ang-(1-7) levels 
Citation: Aritomi S, Niinuma K, Kawakami M, Nakamura T, Konda T, et al. (2012) Cilnidipine, An L-/N-Type Calcium Channel Blocker, Changes the Circulating Angiotensin-(1-7)/Angiotensin II Ratio. J Hypertens 1:102. doi:10.4172/2167-1095.1000102

Page 3 of 6

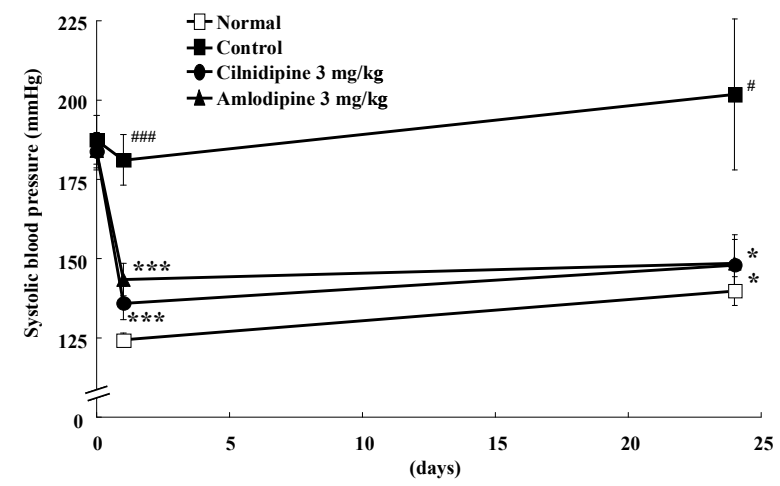

B

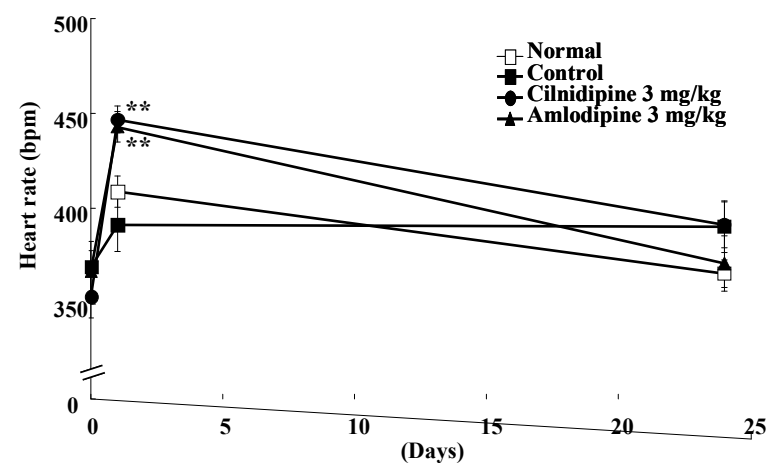

Figure 1: Effects of cilnidipine and amlodipine on systolic blood pressure (A) and heart rate (B) in Sprague-Dawley rats treated with Ang II for 4 weeks. Values are means \pm SEM. ${ }^{\#} P<0.05$, and ${ }^{\# \#} P<0.001$ vs normal group, ${ }^{*} P<$ $0.05,{ }^{* *} P<0.01$, and ${ }^{* * *} P<0.001$ calcium-channel blocker $(\mathrm{CCB})$ vs control group.

\begin{tabular}{|c|c|c|c|c|}
\hline & Normotension & \multicolumn{3}{|c|}{ Hypertension } \\
\cline { 2 - 5 } & $(\mathrm{n}=8)$ & $\begin{array}{c}\text { Control } \\
(\mathrm{n}=8)\end{array}$ & $\begin{array}{c}\text { Cilnidipine } \\
(\mathrm{n}=8)\end{array}$ & $\begin{array}{c}\text { Amlodipine } \\
(\mathrm{n}=8)\end{array}$ \\
\hline Body weight $(\mathrm{g})$ & $382 \pm 10$ & $310 \pm 17^{\dagger \dagger}$ & $344 \pm 8$ & $340 \pm 13$ \\
\hline Heart weight (\%) & $0.26 \pm 0.03$ & $0.34 \pm 0.01^{\dagger+\dagger}$ & $0.31 \pm 0.01$ & $0.31 \pm 0.01$ \\
\hline $\begin{array}{c}\text { Kidney weight } \\
(\%)\end{array}$ & $0.61 \pm 0.04$ & $0.68 \pm 0.03^{\dagger}$ & $0.65 \pm 0.02$ & $0.65 \pm 0.02$ \\
\hline BUN (mg/dL) & $19.6 \pm 0.6$ & $25.4 \pm 2.1^{\dagger}$ & $19.6 \pm 1.1^{*}$ & $19.1 \pm 1.2^{*}$ \\
\hline $\begin{array}{c}\text { Creatinine } \\
(\mathrm{mg} / \mathrm{dL})\end{array}$ & $0.25 \pm 0.01$ & $0.28 \pm 0.01$ & $0.27 \pm 0.01$ & $0.25 \pm 0.01$ \\
\hline $\begin{array}{c}\text { BNP (pg/mL) } \\
\text { Plasma NA } \\
(\mathrm{ng} / \mathrm{mL})\end{array}$ & $57 \pm 5.00$ & $95 \pm 19$ & $72 \pm 12$ & $91 \pm 20$ \\
\hline plasma A (ng/mL) & $5.2 \pm 1.20$ & $5.5 \pm 0.9$ & $3.0 \pm 0.3$ & $3.0 \pm 0.6$ \\
\hline
\end{tabular}

Values presented are the mean \pm SEM. ${ }^{\dagger} \mathrm{P}<0.05,{ }^{+\dagger} \mathrm{P}<0.01,{ }^{\mathrm{t}+\mathrm{P}}<0.001$ vs normotension, ${ }^{*} \mathrm{P}<0.05,{ }^{* *} \mathrm{P}<0.01,{ }^{* * *} \mathrm{P}<0.001$ vs control.Control, vehicle-treated hypertension rats Cilnidipine, cilnidipine $3 \mathrm{mg} / \mathrm{kg}$ treted hypertension rats treated with cilnidipine $3 \mathrm{mg} /$ $\mathrm{kg}$; Amlodipine, hypertension rats treated with amlodipine $3 \mathrm{mg} / \mathrm{kg}$.

Tabel 1: Blood pressure, body weight, and relative organ weight in normotension and hypertension with/without CCB

between the control group and the CCB treatment groups (Figure 2A). As for the effects of the CCBs, the amlodipine-treated group showed a significantly lower Ang-(1-7)/Ang I ratio than the control group (0.7 \pm 0.1 vs $1.1 \pm 0.1, P<0.05$; Figure $2 \mathrm{~B})$, and the cilnidipine-treated group showed a significantly higher Ang-(1-7)/Ang II ratio than the control group ( $3.3 \pm 0.4$ vs $1.8 \pm 0.1, P<0.01$; Figure $2 \mathrm{~B})$.

\section{Gene Expression in the left ventricle}

Gene expression levels of $\mathrm{ACE}, \mathrm{ACE} 2$, and angiotensinogen in the control group were significantly lower than those in the normal group $(P<0.001$ each, Table 2$)$, and BNP expression levels in the control group were significantly higher than those in the normal group $(P<0.01$, Table 2$)$. As for the CCB treatment groups, cilnidipine and amlodipine significantly increased gene expression levels of ACE2 and ACE (cilnidipine, $P<0.01$ and $P<0.01$; amlodipine, $P<0.05$ and $P$ $<0.001$; Table 2). However, amlodipine significantly decreased the ACE2/ACE expression ratio and increased angiotensinogen gene expression levels compared to those in the control group $(P<0.05$, Table 2). The BNP gene expression levels in the control group were significantly higher than those in the normal group $(P<0.01$, Table 2 ). The effects of CCBs were comparable among Ang II-infused groups, with the cilnidipine and amlodipine treatment groups showing significantly lower BNP gene expression levels than the control group $(P<0.01$ and $P<0.05$, Table 2$)$.

\section{PRA and plasma aldosterone levels}

There were no differences in PRA between the control group and the $\mathrm{CCB}$ treatment groups (Figure $3 \mathrm{~A}$ ). However, only cilnidipine significantly decreased plasma aldosterone levels in comparison with the control group $(2.0 \pm 0.4 \mathrm{ng} / \mathrm{mL}$ vs $8.7 \pm 1.3 \mathrm{ng} / \mathrm{mL}, P<0.001$; Figure $3 \mathrm{~B})$.

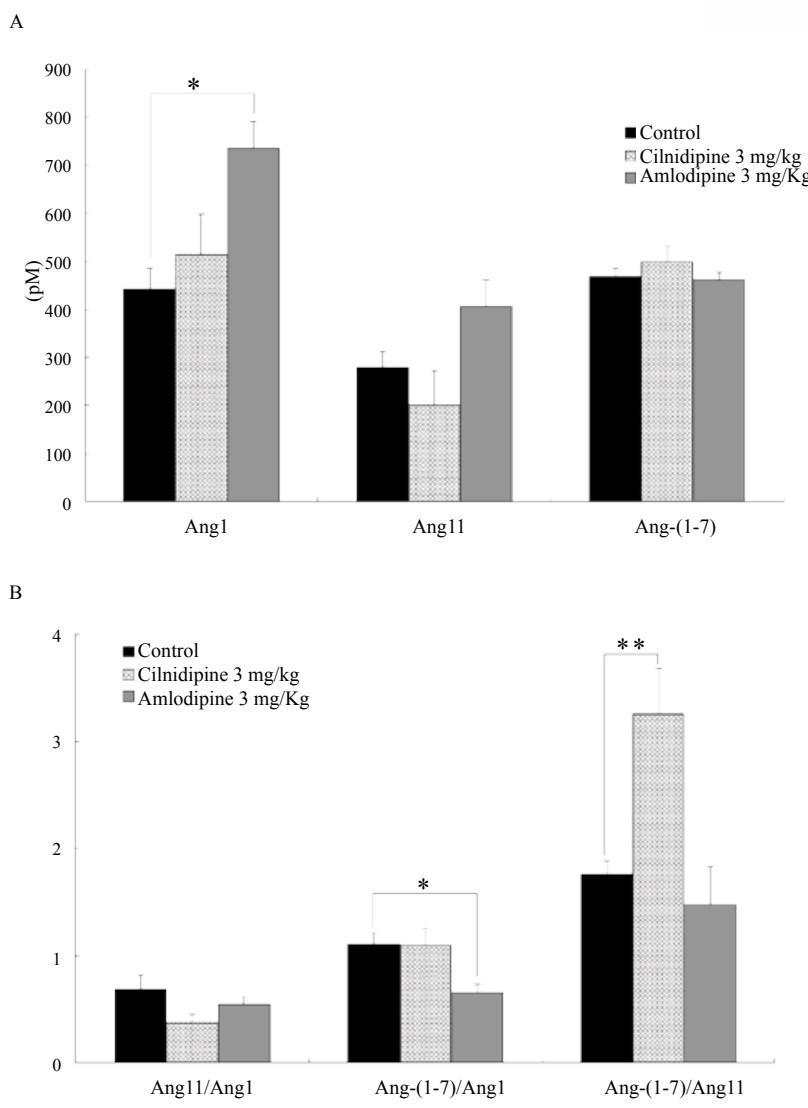

Figure 2: Effects of cilnidipine and amlodipine on circulating levels of angiotensin peptide $(A)$ and the ratios between angiotensins $(B)$ in SpragueDawley rats treated with Ang II for 4 weeks. Values are means \pm SEM. ${ }^{*} P<$ $0.05,{ }^{* *} P<0.01$, and ${ }^{* * *} P<0.001$ calcium-channel blocker (CCB) vs control group. Ang I: Angiotensin I; Ang II: Angiotensin II; Ang-(1-7): Angiotensin-(1-7). 
Citation: Aritomi S, Niinuma K, Kawakami M, Nakamura T, Konda T, et al. (2012) Cilnidipine, An L-/N-Type Calcium Channel Blocker, Changes the Circulating Angiotensin-(1-7)/Angiotensin II Ratio. J Hypertens 1:102. doi:10.4172/2167-1095.1000102

Page 4 of 6

\begin{tabular}{|l|c|c|c|c|}
\hline & Normotension & \multicolumn{3}{|c|}{ Hypertension } \\
\cline { 2 - 5 } & $(\mathrm{n}=8)$ & $\begin{array}{c}\text { Control } \\
(\mathrm{n}=8)\end{array}$ & $\begin{array}{c}\text { Cilnidipine } \\
(\mathrm{n}=8)\end{array}$ & $\begin{array}{c}\text { Amlodipine } \\
(\mathrm{n}=8)\end{array}$ \\
\hline ACE & $25 \pm 4$ & $9 \pm 1^{\dagger+\dagger}$ & $21 \pm 2^{\star *}$ & $24 \pm 1^{* * *}$ \\
\hline ACE2 & $4.0 \pm 0.3$ & $2.1 \pm 0.2^{\dagger+\dagger}$ & $3.7 \pm 0.5^{\star *}$ & $3.3 \pm 0.3^{*}$ \\
\hline ACE2/ACE & $0.19 \pm 0.03$ & $0.25 \pm 0.03$ & $0.18 \pm 0.02$ & $0.14 \pm 0.01^{*}$ \\
\hline Angiotensinogen & $0.26 \pm 0.03$ & $0.15 \pm 0.02^{\dagger}$ & $0.26 \pm 0.03$ & $0.31 \pm 0.03^{* *}$ \\
\hline BNP & $92 \pm 23$ & $224 \pm 28$ & $94 \pm 21^{* *}$ & $95 \pm 7^{*}$ \\
\hline
\end{tabular}

Values presented are the mean \pm SEM. ${ }^{+} \mathrm{P}<0.05$, ${ }^{\mathrm{t}+\mathrm{P}} \mathrm{P}<0.001$ vs normotension, ${ }^{*} \mathrm{P}<0.05,{ }^{* *} \mathrm{P}<0.01,{ }^{* * *} \mathrm{P}<0.001$ vs control.Control, vehicle-treated hypertension rats; Cilnidipine, cilnidipine $3 \mathrm{mg} / \mathrm{kg}$ treted hypertension rats treated with cilnidipine $3 \mathrm{mg} / \mathrm{kg}$; Amlodipine, hypertension rats treated with amlodipine $3 \mathrm{mg} / \mathrm{kg}$.

Tabel 2: RT-qPCR of ACE, ACE2, angiotensinogen and BNP in the left ventricular.

A

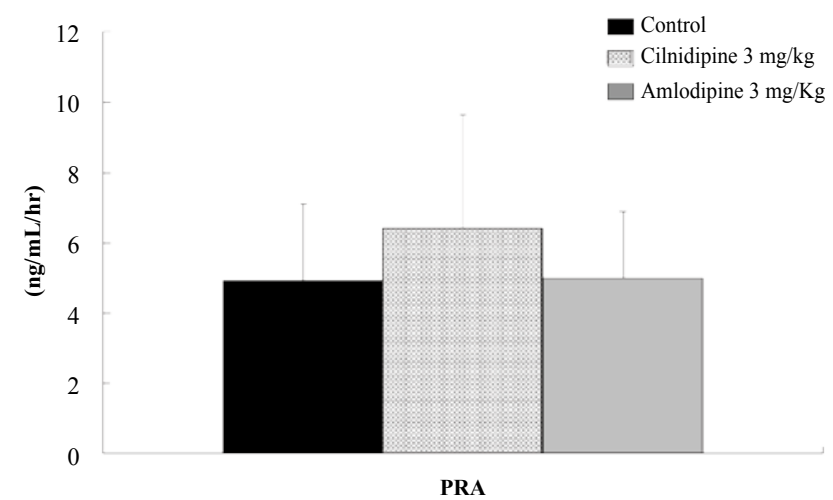

B

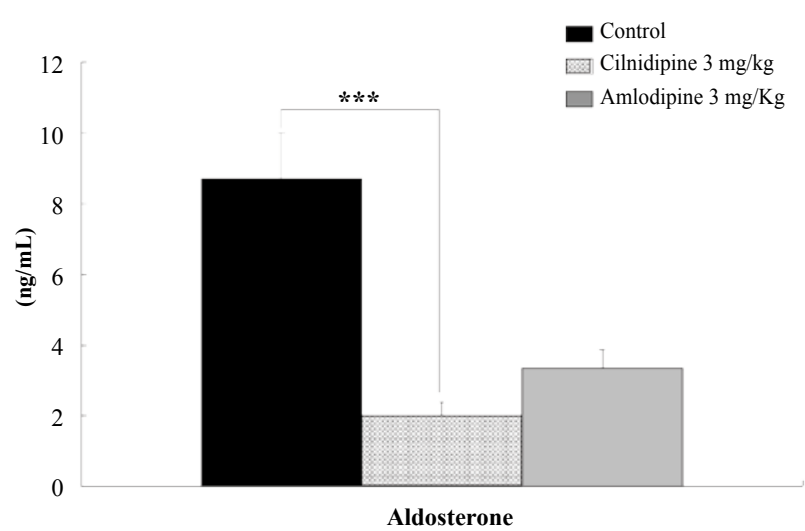

Figure 3: Effects of cilnidipine and amlodipine on plasma renin activity (A), and plasma aldosterone levels $(B)$ in Sprague-Dawley rats treated with Ang II for 4 weeks. Values are means \pm SEM. ${ }^{* \star \star} P<0.001$ CCB vs control group.

\section{Cardiac fibrosis}

The cardiac fibrosis area in the control group was significantly higher than that in the normal group $(6.4 \% \pm 0.4 \%$ vs $3.8 \% \pm 0.2 \%$, $P<0.001$; Figure 4A). The cilnidipine-treated groups exhibited a clear declining trend, and only $10 \mathrm{mg} / \mathrm{kg}$ of cilnidipine succeeded in suppressing the expansion of the area of cardiac fibrosis $(4.7 \% \pm 0.3 \%$, $P<0.05$; Figure $4 \mathrm{~A})$.

\section{Discussion}

In our study, cilnidipine raised the Ang-(1-7)-Ang II ratios in the Ang II-infused hypertensive model. This finding implied that cilnidipine might tilt the balance between the 2 arms of the RAS from vasoconstriction (ACE-Ang II-AT1R) to vasodilatation [ACE2-Ang(1-7)-Mas receptor]. According to a recent novel concept, the final functional effects of the RAS may reflect the balance between these $2 \mathrm{arms}$ [6-8]. Circulating RAS is well recognized for its role in hemodynamic regulation through Ang II, which is a potent vasoconstrictor, and the counterregulatory peptide, Ang-(1-7), which is a vasodilator. Moreover, in diabetic nephropathy, the activity of the ACE-Ang II-AT1R arm mainly leads to exacerbation of the disease, and the ACE2-Ang-(17)-Mas receptor arm mainly acts as a counterregulatory mechanism. Although all major components of the RAAS exhibit profibrotic activity, Ang II appears to be the dominant hormone that is responsible for cardiac fibrosis in hypertensive heart disease [22]. Ang II, which is produced locally by activated macrophages and fibroblasts, is thought to exert its effects by directly inducing nicotinamide adenine dinucleotide phosphate oxidase activity, stimulating transforming growth factor- $\beta 1$ production, and triggering fibroblast proliferation and differentiation into collagen-secreting myofibroblasts $[23,24]$. Taken together, the above results suggest that cilnidipine has suppressive effects on fibrosis through the tilting of the balance between the 2 arms of the RAS from vasoconstriction (ACE-Ang II-AT1R) to vasodilatation.

The PRA levels of this hypertensive model were markedly suppressed by the Ang II-induced negative feedback effects on renin release [25], and CCBs, at least at this dosage, had no effect on Ang II-induced negative feedback. However, amlodipine caused a 1.7-fold increase in the circulating levels of Ang I that were regulated by PRA. The reason why amlodipine increased Ang I levels is that amlodipine may increase the production of angiotensinogen because amlodipine caused a 2.1-fold increase in the expression levels of angiotensinogen.

As for the gene expression of ACE and ACE2, Ang II suppressed

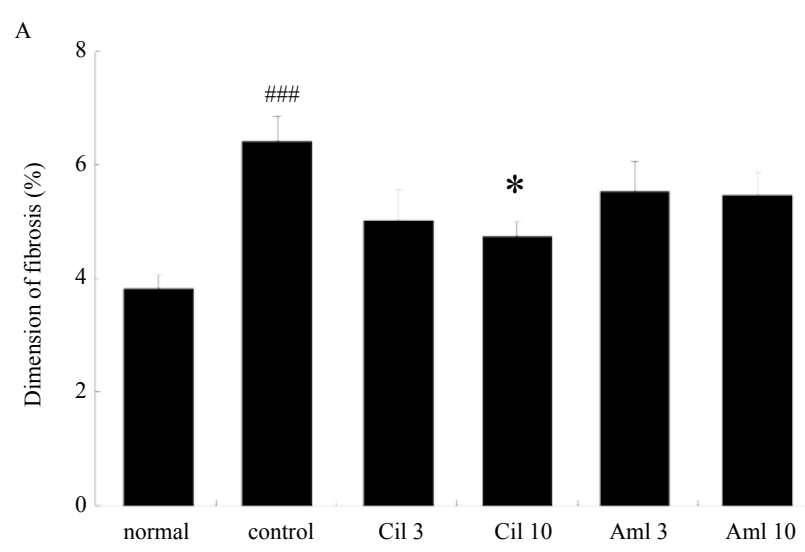

B

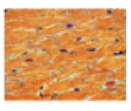

normal

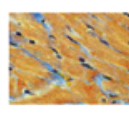

control

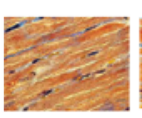

Cil 3

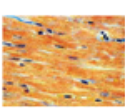

Cil 10

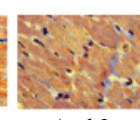

$\mathrm{Aml} 3$

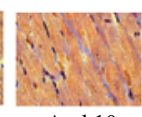

Aml 10
Figure 4: Myocardial fibrosis (A) and Masson's trichrome stained sections (B) in Sprague-Dawley rats treated with Ang II for 4 weeks. Values are means \pm SEM. ${ }^{\# \#} P<0.001$ vs normal group, ${ }^{*} P<0.05$ vs control group. Cil 3 cilnidipine $3 \mathrm{mg} / \mathrm{kg}$; Cil 10: cilnidipine $10 \mathrm{mg} / \mathrm{kg}$; Aml 3: amlodipine $3 \mathrm{mg} / \mathrm{kg}$; Aml 10: amlodipine $10 \mathrm{mg} / \mathrm{kg}$. 
the expression levels of these genes in the left ventricle. Furthermore, CCBs increased the expression levels of the ACE gene through their antihypertensive effects, and only cilnidipine ameliorated the expression levels of the ACE2 gene. This may be attributable to differences in aldosterone suppressive effects between cilnidipine and amlodipine. It has already been reported that aldosterone regulates the levels of expression of these genes in cultured rat cardiomyocytes [26], and cilnidipine suppressed plasma aldosterone levels despite the failure of amlodipine to exert the same effect [13]. In this study, both cilnidipine and amlodipine suppressed the increases in plasma aldosterone levels, but the aldosterone levels of the amlodipine group were about 1.7 times higher that of the cilnidipine group. This difference may partially contribute to the differentiation of ACE/ACE2 gene expression. Furthermore, it has been suggested that the suppression of sympathetic nerve activity by the drug induces the expression of ACE2 [27], and cilnidipine is a unique CCB with inhibitory actions on sympathetic N-type calcium channels. In addition, the drug has been demonstrated to exert anti-sympathetic actions in various examinations from cells to humans [9]. These reports suggest that the inhibitory effects of cilnidipine on sympathetic nerve activity induce ACE2 expression. Thus, further study will be necessary to determine the effects of cilnidipine on ACE2. However this study is insufficient for the accurate assessment of the balance of Ang-(1-7)/Ang II due to artifact of angiotensinogen. Thus to evaluate changes in the expression of Ang-(1-7)/Ang II directly, in vitro study may be necessary to confirm the present findings, because the in vivo experiments may be influenced by many factors that modulate the heart condition.

In conclusion, the results of the present study demonstrated that the L-/N-type CCB cilnidipine changed the balance between Ang(1-7) and Ang II and increased the proportion of plasma Ang-(1-7). There is accumulating evidence to show that stimulation of the ACE2Ang-(1-7)-Mas arm yields cardioprotective and antihypertensive effects [28-30], which raises the possibility that cilnidipine also exerts cardioprotective activity through increases in the activity of this arm.

\section{References}

1. Huentelman MJ, Zubcevic J, Katovich MJ, Raizada MK (2004) Cloning and characterization of a secreted form of angiotensin-converting enzyme 2. Regul Pept 122: 61-67

2. Chappell MC, Modrall JG, Diz DI, Ferrario CM (2004) Novel aspects of the renal renin-angiotensin system: angiotensin-(1-7), ACE2 and blood pressure regulation. Contrib Nephrol 143: 77-89.

3. Ferrario CM (2003) Contribution of angiotensin-(1-7) to cardiovascular physiology and pathology. Curr Hypertens Rep 5: 129-134.

4. Ferrario CM, Chappell MC (2004) Novel angiotensin peptides. Cell Mol Life Sci 61: $2720-2727$.

5. Iyer SN, Chappell MC, Averill DB, Diz DI, Ferrario CM (1998) Vasodepressor actions of angiotensin-(1-7) unmasked during combined treatment with lisinopril and losartan. Hypertension 31: 699-705.

6. Simoes e Silva AC, Pinheiro SV, Pereira RM, Ferreira AJ,Santos RA (2006) The Therapeutic potential of Angiotensin-(1-7) as a novel Renin-Angiotensin System mediator. Mini Rev Med Chem 6: 603-609.

7. Matsui T, Tamaya K, Matsumoto K, Osajima Y, Uezono K, et al. (1999) Plasma concentrations of angiotensin metabolites in young male normotensive and mild hypertensive subjects. Hypertens Res 22: 273-277.

8. Nogueira Al, Souza Santos RA, Simoes e Silva AC, Cabral AC, Vieira RL, et al (2007) The pregnancy-induced increase of plasma angiotensin-(1-7) is blunted in gestational diabetes. Regul Pept 141: 55-60.

9. Takahara A (2009) Cilnidipine: A new generation Ca channel blocker with inhibitory action on sympathetic neurotransmitter release. Cardiovasc Ther 27 124-39.
10. Takahara A, Koganei H, Takeda T, Iwata S (2002) Antisympathetic and hemodynamic property of a dual $\mathrm{L} / \mathrm{N}$-type $\mathrm{Ca}(2+)$ channel blocker cilnidipine in rats. Eur J Pharmacol 434: 43-47.

11. Shiga T, Yamada Y, Matsuda N, Tanaka T, Urae A, et al. (2007) Influence of cilnidipine or nisoldipine on sympathetic activity in healthy male subjects. Heart Vessels 22: 404-409.

12. Nagahama S, Norimatsu T, Maki T, Yasuda M, Tanaka S (2007) The effect of combination therapy with an L/N-Type $\mathrm{Ca}(2+)$ channel blocker, cilnidipine and an angiotensin II receptor blocker on the blood pressure and heart rate in Japanese hypertensive patients: an observational study conducted in Japan. Hypertens Res 30: 815-822.

13. Konda T, Enomoto A, Aritomi S, Niinuma K, Koganei H, et al. (2009) Differen effects of L/N-Type and L-Type calcium channel blockers on the reninangiotensin-aldosterone system in SHR/Izm. Am J Nephrol 30: 155-161.

14. Takahara A, Nakamura $Y$, Wagatsuma H, Aritomi S, Nakayama A, et al. (2009) Long-term blockade of $\mathrm{L} / \mathrm{N}$-type $\mathrm{Ca}(2+)$ channels by cilnidipine ameliorates repolarization abnormality of the canine hypertrophied heart. $\mathrm{Br} \mathrm{J}$ Pharmacol 158: $1366-1374$

15. Fournier A, Oprisiu-Fournier R, Serot JM, Godefroy O, Achard JM, et al. (2009) Prevention of dementia by antihypertensive drugs: how AT1-receptor-blockers and dihydropyridines better prevent dementia in hypertensive patients than thiazides and ACE-inhibitors. Expert Rev Neurother 9: 1413-1431.

16. Aritomi S, Niinuma K, Ogawa T, Konda T, Nitta K (2011) The effects of a combination of the calcium channel blocker Cilnidipine and the AT1 receptor antagonist Valsartan on the angiotensin II-renin feedback interruption. Am J Nephrol 33: 168-175.

17. Aritomi S, Koganei H, Wagatsuma H, Mitsui A, Ogawa T, et al. (2010) The $\mathrm{N}$-type and L-type calcium channel blocker cilnidipine suppresses renal injury in Dahl rats fed a high-salt diet. Heart Vessels 25: 549-555.

18. Konoshita T, Makino Y, Kimura T, Fujii M, Wakahara S, et al. (2010) Genomic Disease Outcome Consortium Study Investigators. A new-generation N/L-type calcium channel blocker leads to less activation of the renin-angiotensin system compared with conventional L type calcium channel blocker. J Hypertens 28 2156-2160.

19. Fujita T, Ando K, Nishimura H, Ideura T, Yasuda G, et al. (2007) Cilnidipine versus Amlodipine Randomised Trial for Evaluation in Renal Desease(CARTER) Study Investigators. Antiproteinuric effect of the calcium channel blocker cilnidipine added to renin-angiotensin inhibition in hypertensive patients with chronic renal disease. Kidney Int 72: 1543-1549.

20. Shao W, Seth DM, Navar LG (2010) Angiotensin II type 1 receptor-mediated augmentation of urinary excretion of endogenous angiotensin II in Val5angiotensin II-infused rats. Hypertension 56: 378-383.

21. Shao W, Seth DM, Navar LG (2009) Augmentation of endogenous intrarenal angiotensin II levels in Val5-ANG II-infused rats. Am J Physiol Renal Physio 296: F1067-F1071.

22. Watanabe T, Barker TA, Berk BC (2005) Angiotensin II and the endothelium: Diverse signals and effects. Hypertension 45: 163-169.

23. Rosenkranz S (2004) TGF- $\beta 1$ and angiotensin networking in cardiac remodelling. Cardiovasc Res 63: 423-432.

24. Bataller R, Schwabe RF, Choi YH, Yang L, Paik YH, et al. (2003) NADPH oxidase signaltransduces angiotensin II in hepatic stellate cells and is critical in hepatic fibrosis. J Clin Invest 112: 1383-1394.

25. Von Thun AM, El-Dahr SS, Vari RC, Navar LG (1994) Modulation of renninangiotensin and kallikrein gene expression in experimental hypertension. Hypertension 23: 131-136.

26. Yamamuro M, Yoshimura M, Nakayama M, Abe K, Sumida H, et al. (2008) Aldosterone, but not angiotensin II, reduces angiotensin converting enzyme 2 gene expression levels in cultured neonatal rat cardiomyocytes. Circ $\mathrm{J} 72$ 1346-1350.

27. Kar S, Gao L, Zucker IH (2010) Exercise training normalizes ACE and ACE2 in the brain of rabbits with pacing-induced heart failure. J Appl Physiol 108: 923-932.

28. Santos RA, Ferreira AJ, Simoes E Silva AC (2008) Recent advances in the angiotensin-converting enzyme 2-angiotensin(1-7)-Mas axis. Exp Physio 93 519-527. 
Citation: Aritomi S, Niinuma K, Kawakami M, Nakamura T, Konda T, et al. (2012) Cilnidipine, An L-/N-Type Calcium Channel Blocker, Changes the Circulating Angiotensin-(1-7)/Angiotensin II Ratio. J Hypertens 1:102. doi:10.4172/2167-1095.1000102

Page 6 of 6

29. Ferreira AJ, Santos RA, Almeida AP (2001) Angiotensin-(1-7): cardioprotective effect in myocardial ischemia/reperfusion. Hypertension 38: 665-668.

30. Benter IF, Yousif MH, Anim JT, Cojocel C, Diz DI (2006) Angiotensin-(1-7) prevents development of severe hypertension and end-organ damage in spontaneously hypertensive rats treated with L-NAME. Am J Physiol Heart Circ Physiol 290: H684-H691. 